\title{
Effect of Dynamic SGS Model in a Kerosene-LOx Swirl Injector under Supercritical Condition
}

\author{
Jun-Young Heo* and Ji-Seok Hong** \\ Department of Aerospace and Mechanical Engineering, Korea Aerospace University, Goyang 412-791, Korea
}

\section{Hong-Gye Sung***}

School of Aerospace and Mechanical Engineering, Korea Aerospace University, Goyang 412-791, Korea

\begin{abstract}
In this study, numerical simulations are carried out to investigate the dynamic SGS model effects in a Kerosene-LOx coaxial swirl injector under high pressure conditions. The turbulent model is based on large-eddy simulation (LES) with real-fluid transport and thermodynamics. To assess the effect of the dynamic subgrid-scale (SGS) model, the dynamic SGS model is compared with that of the algebraic SGS model. In a swirl injector under supercritical pressure, the characteristics of temporal pressure fluctuation and power spectral density (PSD) present comparable discrepancies dependant on the SGS models, which affect the mixing characteristics. Mixing efficiency and the probability density (PDF) function are conducted for a statistical description of the turbulent flow fields according to the SGS models. The back-scattering of turbulent kinetic energy is estimated in terms of the film thickness of the swirl injector.
\end{abstract}

Key words: Swirl Injector, LES(Large-Eddy Simulation), Supercritical Condition, Dynamic Sub-grid Scale Model

\section{Nomenclature}

$A_{1}, A_{2}, B_{1}, B_{0}, C_{1}, C_{0}$
$a, b$
$c$
$D$
$d$

$d_{1}, d_{2}, d_{3}, d_{4}, d_{5}, d_{6}$ E

$H$

$k$

$M_{w}$ $N_{x}, N_{y}, N_{z}$

$f$ $p$ coefficients for estimation of

$=$ attraction and repulsive factor

$=$ speed of sound

$=$ diffusivity

$=$ functions of critical compressibility factor

$=$ coefficients for estimation of $\delta_{1}$

$=$ specific total energy

$=$ energy flux

= functions of critical compressibility factor

$=$ molecular weight

$=$ the number of grids for each direction

$=$ mixture fraction

$=$ pressure
$Q, q$

$R_{u}$

$S_{p} S_{o}$

$T$

$t$

$U$

$u$

$u$

V

$x$

Z

Greek

$\alpha$

$\delta$

$\delta_{1}, \delta_{2}$

$\Theta$

$\kappa$
$=$ heat flux

$=$ universal gas constant

$=$ swirl number of fuel and oxidizer

$=$ temperature

$=$ time

$=$ diffusion velocity

$=$ velocity

$=$ volume

$=$ spatial coordinate

$=$ compressibility factor

$=$ function for cubic equation of state

$=$ Kronecker delta

$=$ functions of critical compressibility factor

$=$ species diffusive flux

$=$ association parameter
This is an Open Access article distributed under the terms of the Creative Commons Attribution Non-Commercial License (http://creativecommons.org/licenses/by$\mathrm{nc} / 3.0 /$ which permits unrestricted non-commercial use, distribution, and reproduction in any medium, provided the original work is properly cited. (cc) * Post-Doctoral Researcher

** Ph. D Student

*** Professor, Corresponding author: hgsung@kau.ac.kr 


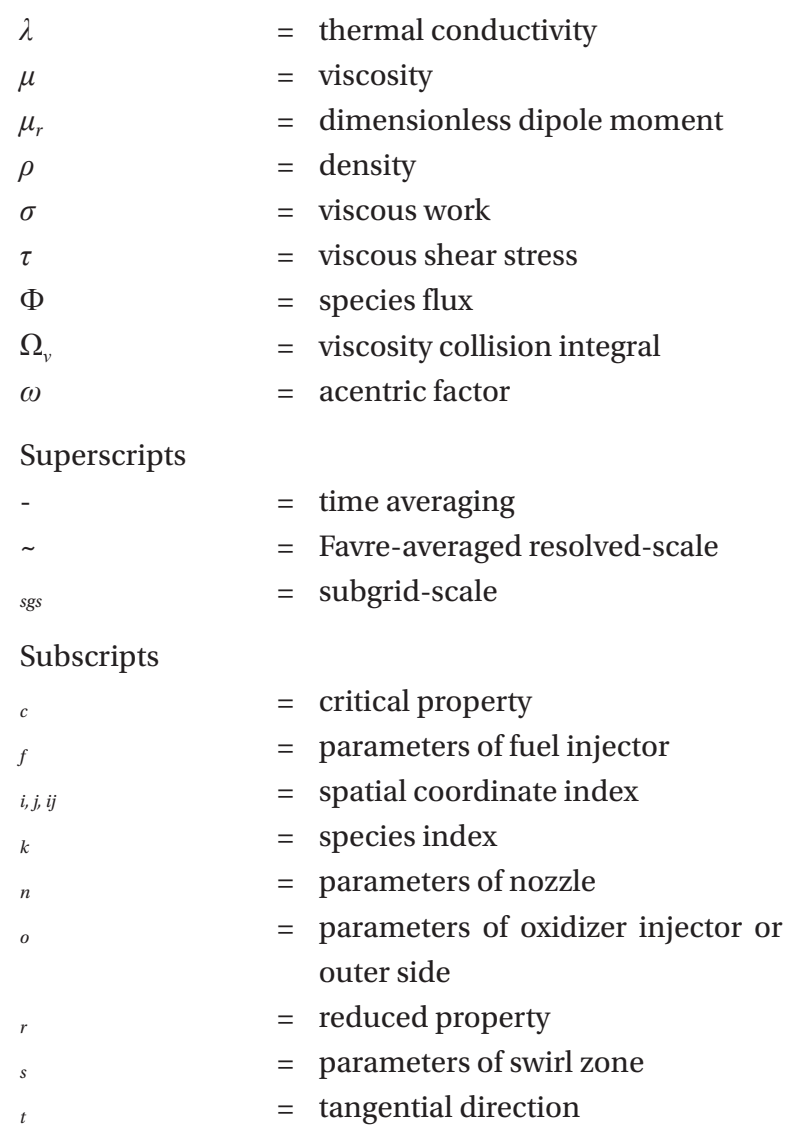

\section{Introduction}

A liquid rocket engine for a space launch vehicle operates at high pressure in order to increase the thrust and combustion efficiency so that its propellants are exposed to supercritical conditions in the combustion chamber. The supercritical condition refers to the state beyond its critical point. In such conditions, the propellants experience the transition of thermo-physical properties differently than those of subcritical conditions. After the liquid propellant is introduced into the injector and combustion chamber, its properties are varied continuously and the distinction between gas and liquid becomes ambiguous. The process goes through a "liquid like $\rightarrow$ gas like" at supercritical pressure, whereas it undergoes "liquid core $\rightarrow$ break up $\rightarrow$ atomization $\rightarrow$ vaporization" at subcritical pressures. Also, as the temperature increases, the thermodynamic properties vary continuously and a distinction between the liquid and gas phase becomes ambiguous under the high pressure condition [1]. Intermolecular forces and the molecular body volume are considered to be important factors in the real fluid. In order to understand the real fluid behavior, effects such as compressibility, van der Waals force, molecular dissociation, and non-equilibrium thermodynamic quantity must be taken into account. An equation of state capable of handling the behavior of real fluid at a high pressure and low temperature regime is required for real fluid. Therefore, conventional predictions of thermodynamic properties and mixing dynamics become inappropriate in explaining thermodynamic relationships for a wide range of pressures and temperatures which have to employ recent advances in numerical research. The thermo-physical characteristics of liquid oxygen/gaseous hydrogen flame in a shear coaxial injector at supercritical pressure using direct numerical simulation have been investigated by Oefelein [2]. The diffusion dominated mode of combustion and the sharp gradients of thermo-physical properties were identified in the region close to the injector element. Also, the results show that a strong recirculation zone formed near the LOx post and the unsteady stagnation area inside the zone provides the primary flame holding mechanism. Zong and Yang [3] applied a large eddy simulation for the identification of cryogenic fluid dynamics in a swirl injector operating at supercritical pressure. They observed the hydrodynamic instabilities in both the axial and azimuthal directions within the liquid oxygen film and the flow recirculation in the vicinity of the injector exit. This provided insight about the flow dynamics in the LRE swirl injector. Giorgi and Leuzzi [4] performed a numerical analysis of mixing and combustion in a liquid oxygen/gaseous methane shear coaxial injector at supercritical conditions. The study focused on the importance of the real fluid effect. Wang et al. [5] studied the convective heat transfer effect in a flowfield of cryogenic fluid under supercritical pressure. The study showed that the conventional approach cannot be used for the prediction of heat transfer at supercritical conditions.

Aturbulencestructure with turbulent energy is represented by the vorticity, and the vortex structure at a high flow speed causes a wide range of eddy sizes. The large eddy is generated by the turbulence energy, the turbulent energy cascade occurred from large eddy to small eddy. Since the small eddy is easily dissipated, the turbulence energy vanished. When using the time averaging technique, the turbulence modeling will be required in the overall turbulence region. However, for each problem, the turbulence model needs to adjust the coefficients in order to increase the simulation accuracy. To overcome these defects, the eddy which is larger than the grid size is computed directly, the small eddy is calculated by the SGS (sub-grid scale) model for improving the accuracy of the turbulent flow; LES (LargeEddy Simulation). Since the energy dissipation occurred by small eddies, the SGS modeling is an important part of the LES. Most of the SGS models are based on the eddy-viscosity 
type model. The algebraic Smagorinsky model proposed by Lilly $[6,7]$ is the most widely used in LES. The eddy viscosity is calculated algebraically to simplify the equation, and it is based on the equilibrium hypothesis. Germano et al. [8] pointed out that the algebraic model has several limitations. First of all, the algebraic model can't capture the backscatter of energy, which refers to the backward turbulent energy occurring from the small eddy to large eddy. Because this phenomenon is important in the transition region, they proposed a dynamic subgrid-scale eddy viscosity model to improve this deficiency. The dynamic model calculates the SGS model coefficients using the information of the resolved flow field during the simulation. Many researchers have studied this dynamic concept across a wide field of research. Jaberi and Colucci [9] presented localized SGS models in turbulent flows. The statistics such as PDF (probability density function) of the resolved field in LES are compared with results simulated in DNS. Jammalamadaka et al. [10] studied the effect of the subgrid-scale stress term on the velocity field and shock-dependence. A priori and a posteriori were also conducted to indicate the new dynamic model. Urzay et al. [11] studied the dynamics of the backscatter of kinetic energy in high-speed turbulent reacting flows. The effects of compressibility and combustion according to the SGS backscatter of kinetic energy were analyzed.

In this study, a comparison and analysis of the mixing characteristics are conducted in accordance with sub-grid scale models to take account of the effect of the turbulence viscosity. It is also investigated how the dissimilarity between the predicted results affects the characteristics of the flow structure. Power spectral densities of pressure fluctuations are also analyzed to evaluate the instability caused by pressure oscillation in a swirl injector at the supercritical condition.

\section{Numerical Method}

\subsection{Filtered Transport Equations}

The theoretical formulation is based on the filtered Favre averaged mass, momentum, energy, and mixture fraction conservation equations in Cartesian coordinates. Turbulent closure is achieved by using a Large-eddy Simulation (LES) in which large scale turbulent structures are directly computed and the small scale structures are treated with the analytic or empirical modeling. The governing equations can be written as:

$$
\frac{\partial \bar{\rho}}{\partial t}+\frac{\partial \bar{\rho} \tilde{u}_{j}}{\partial x_{j}}=0
$$

$$
\begin{aligned}
& \frac{\partial \bar{\rho} \tilde{u}_{i}}{\partial t}+\frac{\partial\left(\bar{\rho} \tilde{u}_{i} \tilde{u}_{j}+\bar{p} \delta_{i j}\right)}{\partial x_{j}}=\frac{\partial\left(\bar{\tau}_{i j}-\tau_{i j}^{s g s}+D_{i j}^{s g s}\right)}{\partial x_{j}} \\
& \frac{\partial \bar{\rho} \tilde{E}}{\partial t}+\frac{\partial\left[(\bar{\rho} \tilde{E}+\bar{p}) \tilde{u}_{i}\right]}{\partial x_{i}}=\frac{\partial}{\partial x_{i}}\left(\bar{q}_{i}+\tilde{u}_{j} \bar{\tau}_{i j}-Q_{i}^{s g s}-H_{i}^{s g s}+\sigma_{i j}^{s g s}\right) \text { (3) } \\
& \frac{\partial \bar{\rho} \tilde{f}}{\partial t}+\frac{\partial\left(\bar{\rho} \tilde{u}_{i} \tilde{f}\right)}{\partial x_{i}}=\frac{\partial}{\partial x_{i}}\left(\bar{\rho} \tilde{D} \frac{\partial \tilde{f}}{\partial x_{i}}+\Phi_{i}^{s g s}\right)
\end{aligned}
$$

The unclosed sub-grid scale terms are defined as:

$$
\begin{aligned}
& \tau_{i j}^{s g s}=\bar{\rho}\left(u_{i} u_{j}-\tilde{u}_{i} \tilde{u}_{j}\right) \\
& D_{i j}^{s g s}=\left(\bar{\tau}_{i j}-\tilde{\tau}_{i j}\right) \\
& Q_{i}^{s g s}=\left(\bar{q}_{i j}-\tilde{q}_{i j}\right) \\
& H_{i}^{s g s}=\bar{\rho}\left(E u_{i}-E \tilde{u}_{i}\right)+\left(\overline{p u_{i}}-\bar{p} \tilde{u}_{i}\right) \\
& \sigma_{i}^{s g s}=\left(\overline{u_{j} \tau_{i j}}-\tilde{u}_{j} \tilde{\tau}_{i j}\right)
\end{aligned}
$$$$
\Phi_{i}^{s g s}=\bar{\rho}\left(u_{i} f-\tilde{u}_{i} \tilde{f}\right)
$$

$\tau_{i j}^{s g s}, D_{i j}{ }^{s g s}, Q_{i}^{s g s}, H_{i}^{s g s}, \sigma_{i}^{s g s}$ and $\Phi_{i}^{s g s}$ are the SGS stress, nonlinearity of viscous stress term, heat flux, energy flux, viscous work, and conserved scalar flux, respectively.

In the algebraic Smagorinsky model, the SGS stress is represented by a constant eddy viscosity to simplify the equation,

$$
\tau_{i j}^{s g s}-\frac{\delta_{i j}}{3} \tau_{k k}^{s g s}=-2\left(C_{R} \bar{\Delta}\right)^{2}|\tilde{S}|
$$

where the quantity $C_{R}$ represents the compressible Smagorinsky constant.

The dynamic Smagorinsky model suggested by Germano [8] proposes the subgrid stress $\tau_{i j}^{s g s}$ and subtest stress $T_{i j}$ as following:

$$
\begin{gathered}
\tau_{i j}^{s g s}-\frac{\delta_{i j}}{3} \tau_{k k}^{s g s}=-2 C_{R} \bar{\Delta}^{2} \bar{\rho}|\tilde{S}|\left(\tilde{S}_{i j}-\frac{\delta_{i j}}{3} \tilde{S}_{l l}\right)=C_{R} \alpha_{i j} \\
T_{i j}-\frac{\delta_{i j}}{3} T_{k k}=-2 C_{R} \hat{\Delta}^{2} \hat{\bar{\rho}}|\tilde{\tilde{S}}|\left(\tilde{\tilde{S}}_{i j}-\frac{\delta_{i j}}{3} \tilde{\tilde{S}}_{l l}\right)=C_{R} \beta_{i j}
\end{gathered}
$$

where

$$
\begin{aligned}
& \alpha_{i j}=-2 \bar{\Delta}^{2} \bar{\rho}|\tilde{S}|\left(\tilde{S}_{i j}-\frac{\delta_{i j}}{3} \tilde{S}_{l l}\right) \\
& \beta_{i j}=-2 \hat{\Delta}^{2} \hat{\bar{\rho}}|\tilde{\tilde{S}}|\left(\widetilde{\tilde{S}}_{i j}-\frac{\delta_{i j}}{3} \widetilde{\tilde{S}}_{l l}\right)
\end{aligned}
$$


The resolved turbulent stresses, $L_{i j}$, are defined as:

$$
\begin{aligned}
& L_{i j}=C_{R}\left(\beta_{i j}-\hat{\alpha}_{i j}\right)+\frac{\delta_{i j}}{3}\left(T_{k k}-\hat{\tau}_{k k}^{s s s}\right)=C_{R} M_{i j}+\frac{\delta_{i j}}{3} L_{k k} \\
& M_{i j}=\beta_{i j}-\hat{\alpha}_{i j}
\end{aligned}
$$

The model coefficient, $C_{R}$, can be dynamically obtained as suggested by Lilly [12] with a least square method in order to minimize the error:

$$
C_{R}=\frac{L_{u_{i} u_{j}} M_{u_{i} u_{j}}}{M_{u_{k} u_{l}} M_{u_{k} u_{l}}}
$$

In the transition region, the algebraic SGS model predicts excessive damping of the resolved structures because of using a constant SGS coefficient, $C_{R}$, leading to an incorrect growth rate of the perturbations. A dynamic model was proposed to overcome these deficiencies by locally calculating the SGS coefficient.

\subsection{Fluid Thermodynamics and Transport}

The SRK [13] and PR [14] equations of state are usually used to predict the thermo-physical properties of real-gas. In general, the SRK model has a higher accuracy for low molecular weight compounds while the PR model has a higher accuracy for high molecular weight. When kerosene and liquid oxygen are used as propellants, neither the SRK model nor the PR model may be suitable. The SRK model under-predicts both density and viscosity for kerosene when compared to the result of the principle of ECS. Whereas the PR model over predicts the properties for liquid oxygen and has better accuracy than the SRK model. However, it still under-predicts the properties for kerosene. The RK-PR model's results of both oxygen and kerosene are, however, similar to those of the principle ECS.

Therefore, Redlich-Kwong-Peng-Robinson (RK-PR) [1517] is adopted to predict the properties of kerosene and oxygen. A Chung's method [18] is also implemented for the calculation of transport properties. The RK-PR equation of state includes $\delta_{1}$ and $k$ as functions of each component's critical compressibility factor, $\mathrm{Z}_{C}$. The coefficients related to the terms are represented in Table 1.

$$
\begin{aligned}
& p=\frac{\rho R_{u} T}{M_{w}-b \rho}-\frac{a \alpha \rho^{2}}{\left(M_{w}+\delta_{1} b \rho\right)\left(M_{w}+\delta_{2} b \rho\right)} \\
& a=\left[\frac{3 y^{2}+3 y d+d^{2}+d-1}{(3 y+d-1)^{2}}\right]\left(\frac{R_{u}^{2} T_{c}^{2}}{p_{c}}\right), \\
& b=\left(\frac{1}{3 y+d-1}\right)\left(\frac{R_{u} T_{c}}{p_{c}}\right), \quad \alpha=\left(\frac{3}{2+T_{r}}\right)^{k}
\end{aligned}
$$

$$
\begin{gathered}
\delta_{1}=d_{1}+d_{2}\left(d_{3}-1.168 Z_{c}\right)^{d_{4}}+d_{5}\left(d_{3}-1.168 Z_{c}\right)^{d_{6}}, \quad \delta_{2}=\frac{1-\delta_{1}}{1+\delta_{1}} \\
y=1+\left[2\left(1+\delta_{1}\right)\right]^{\frac{1}{3}}+\left(\frac{4}{1+\delta_{1}}\right)^{\frac{1}{3}}, \quad d=\frac{1+\delta_{1}^{2}}{1+\delta_{1}} \\
k=\left(1.168 A_{1} Z_{c}+A_{0}\right) \omega^{2}+\left(1.168 B_{1} Z_{c}+B_{0}\right) \omega+\left(1.168 C_{1} Z_{c}+C_{0}\right)
\end{gathered}
$$

Transport properties are predicted by Chung's model. Mass diffusivity is evaluated using Fuller's theorem with the Takahashi method [19].

\subsection{Numerical Scheme}

The governing equations are solved by using the finite volume method. The spatial discretization is achieved by employing a fourth order central differencing scheme [17]. The temporal discretization is resolved using a fourth order Runge-Kutta scheme for integration of the real time terms. Pressure decomposition and preconditioning techniques with dual time stepping are applied to circumvent the round-off errors and the convergence difficulties in the momentum equation. The real-gas fluid thermodynamic relation is applied to a preconditioning scheme [20]. The code is paralleled using an MPI library for a more effective calculation [21-23].

Table 1. Coefficients for Estimation of $\delta_{1}$ and $k$

\begin{tabular}{l|l|l|l}
\hline \multicolumn{2}{c|}{$\delta_{1}$} & \multicolumn{2}{c}{$k$} \\
\hline$d_{1}$ & 0.428363 & $A_{1}$ & -2.4407 \\
\hline$d_{2}$ & 18.496215 & $A_{0}$ & 0.0017 \\
\hline$d_{3}$ & 0.338426 & $B_{1}$ & 7.4513 \\
\hline$d_{4}$ & 0.660000 & $B_{0}$ & 1.9681 \\
\hline$d_{5}$ & 789.723105 & $C_{1}$ & 12.504 \\
\hline$d_{6}$ & 2.512392 & $C_{0}$ & -2.7238 \\
\hline
\end{tabular}

\section{Model Description}

Figure 1 shows the coaxial swirl injector geometry as well as the swirl numbers of liquid oxygen, and kerosene, at 1.598 and 6.548, respectively [24]. The injector is composed of a closed-type LOx injector with a cavity, an open-type Kerosene injector. The kerosene and liquid oxygen are injected into the swirl injector through the tangential passage. The recess length is $3.2 \mathrm{~mm}$ (recess number $=1$ ), and at this condition, a mixing layer is touching the tip of the kerosene injector. The geometric parameters of the injector are listed in Table 2.

The thickness of the LOx post tip is $0.6 \mathrm{~mm}$, and the 
diameters of the LOx and kerosene post are 4.1 and $8.1 \mathrm{~mm}$, respectively. The operation condition is listed in Table 3. Fig. 2 shows a computational domain, divided into 45 blocks which are assigned to 45 processors. The total of grid points reaches about 765,000 nodes. The Gaussian turbulent disturbances are applied to the inlet boundary with an intensity of $5 \%$ of the mean velocity.

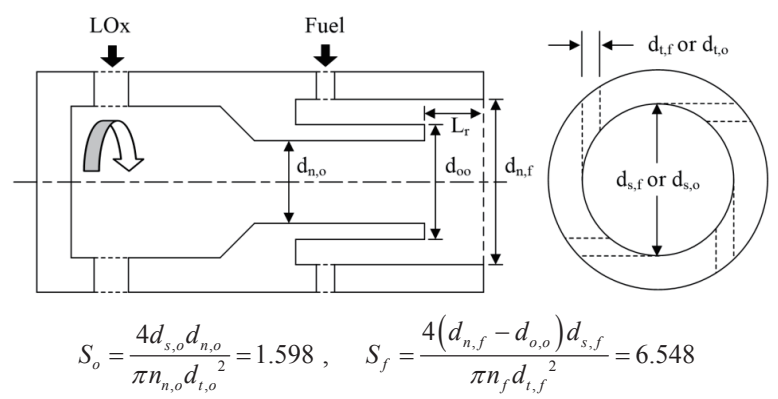

\section{Results}

\subsection{Grid Dependency Test}

To determine the proper grid system, a grid sensitivity analysis has been performed. Three different grid systems, listed in Table 4, are evaluated for validation.

Figure 3 shows the averaged u-velocity contours with stream lines for each grid system. The strong radial pressure gradient by centrifugal force creates an adverse pressure gradient at the exit of the injector, which produces the flow recirculation called the central toroidal recirculation zone (CTRZ). The frequency of the CTRZ is determined by its characteristics of length and velocity [25]. The characteristic length and velocity are defined as a radius of CTRZ and an order of the velocity of CTRZ, respectively.

Fig. 1. Injector Geometry and Swirl Number

Table 2. Geometric Parameters of an Injector, mm

\begin{tabular}{c|c|c|c}
\hline$d_{t, f}$ & 1.05 & $d_{o, o}$ & 5.3 \\
\hline$d_{t, o}$ & 1.75 & $d_{\mathrm{n}, \mathrm{f}}$ & 8.1 \\
\hline$d_{s, \mathrm{f}}$ & 8.1 & $n_{f}$ & 4 \\
\hline$d_{s, o}$ & 7.5 & $n_{o}$ & 8 \\
\hline
\end{tabular}

Table 3. Operation Conditions

\begin{tabular}{|c|c|c|}
\hline \multicolumn{2}{|c|}{ Injector } & Coaxial Swirl \\
\hline \multicolumn{2}{|c|}{ Chamber Pressure } & $100 \mathrm{bar}$ \\
\hline \multicolumn{2}{|c|}{ Fuel } & Kerosene; $350 \mathrm{~K}$ \\
\hline \multicolumn{2}{|c|}{ Oxidizer } & Liquid Oxygen; $103 \mathrm{~K}$ \\
\hline \multirow{2}{*}{ Mass Flow Rate } & Fuel & $0.084 \mathrm{~kg} / \mathrm{s}$ \\
\hline & Oxidizer & $0.232 \mathrm{~kg} / \mathrm{s}$ \\
\hline
\end{tabular}

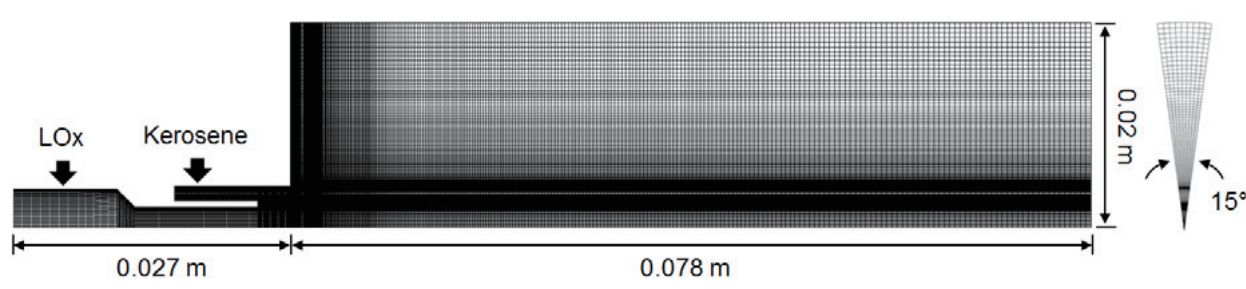

Fig. 2. Computational Domain

Table 4. Grid System

\begin{tabular}{c|c|c|c|c}
\hline & $\mathrm{N}_{\mathrm{x}}$ & $\mathrm{N}_{\mathrm{y}}$ & $\mathrm{N}_{\mathrm{z}}$ & $\left(1 / \mathrm{N}_{\text {total }}\right)^{1 / 3}$ \\
\hline M1 & 25 & 15 & 10 & 0.0640 \\
\hline M2 & 50 & 30 & 10 & 0.0405 \\
\hline M3 & 75 & 45 & 10 & 0.0309 \\
\hline
\end{tabular}




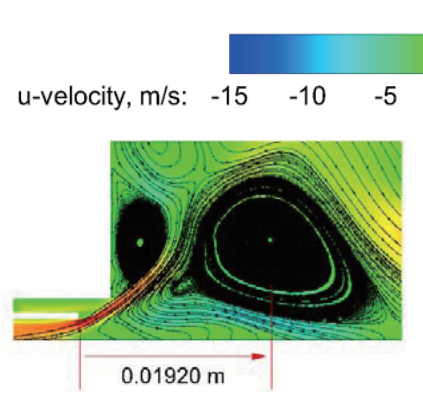

(a) M1

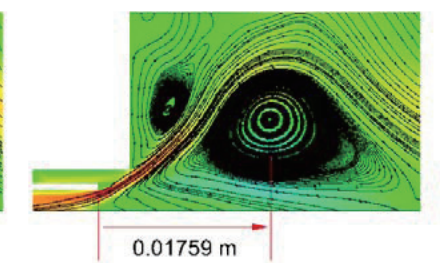

(b) $M 2$
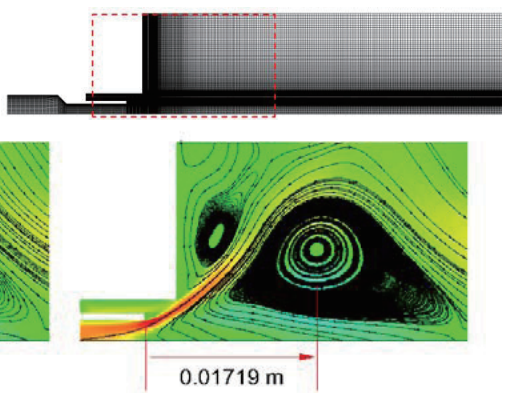

(c) M3

Fig. 3. Averaged u-Velocity Contours with Stream Line for Three Grid Systems

$$
f=\frac{\text { CharacteristicVelocity }}{\text { Characteristic Length }} \approx \frac{10}{0.01} \approx 1 \mathrm{kHz}
$$

In CTRZ, the oscillation pressure induced by the mixing process of fuel and oxygen transfers back to the upstream and affects the wave length inside the oxygen injector. Since the oscillatory flow characteristic plays an important role for determining the flow instabilities of the injector, the frequency is compared to three grid systems. Fig. 4 represents the most dominant frequency at probe 3 shown in Fig. 9. In the case of M1, due to the large size of CTRZ, the excited frequency is lower than that of the higher-

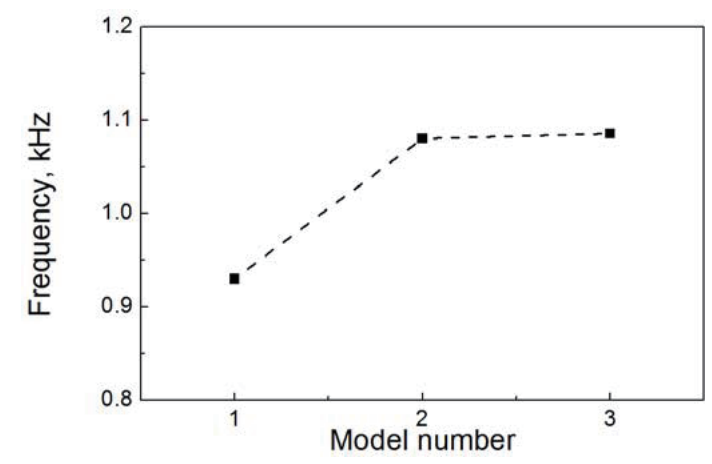

Fig. 4. Comparison of the Most Dominant Frequency at Probe 3

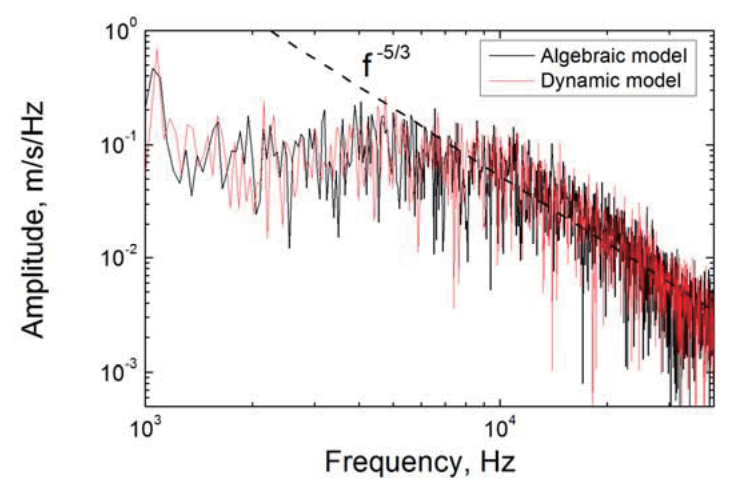

Fig. 5. Frequency Spectra of Turbulent Kinetic Energy for M2 resolution grids, M2 and M3, but the frequencies of M2 and M3 are identical as shown in Fig. 4. Therefore, M2 was selected for this study.

The frequency spectra of turbulent kinetic energy at the mixing layer are shown in Fig. 5. The standard -5/3 law based on the Kolmogorov theory is observed, which supports that the M2 grid system is reasonable.

\subsection{Effects of Dynamic SGS Model}

Figure 6 represents a comparison of the vorticity magnitude obtained by both the algebraic SGS and dynamic SGS models; the algebraic model result is up while the dynamic model result is down.

Both models provide mixing shear layers of Kerosene and LOx, vorticies break up, and a central recirculation zone, which are the general features of swirl injectors, with similar overall structures. The dynamic SGS model has, however, a stronger processing vortex core (PVC), resulting in the larger central toroidal recirculation zone (CTRZ) as shown in Fig. 7. In the dynamic SGS model, the swirling velocity is faster than that of the algebraic SGS model since the dynamic model can capture the local energy generation due to the back scattering effect. Therefore, the size of the CTRZ increases as

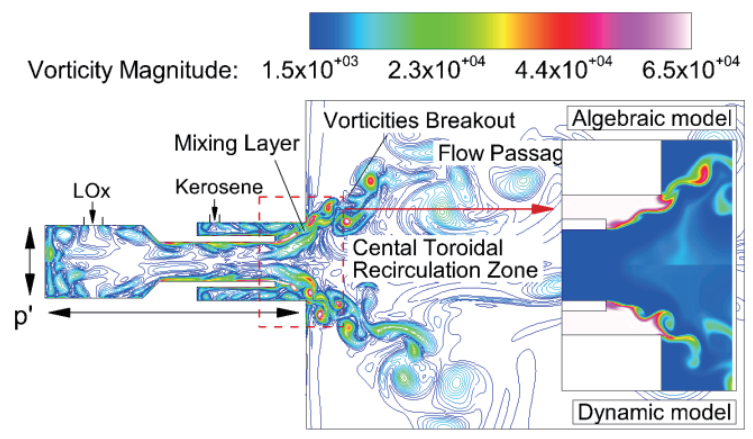

Fig. 6. Vorticity Magnitude of a Swirl Injector; Algebraic Model (up) and Dynamic Model (down) 


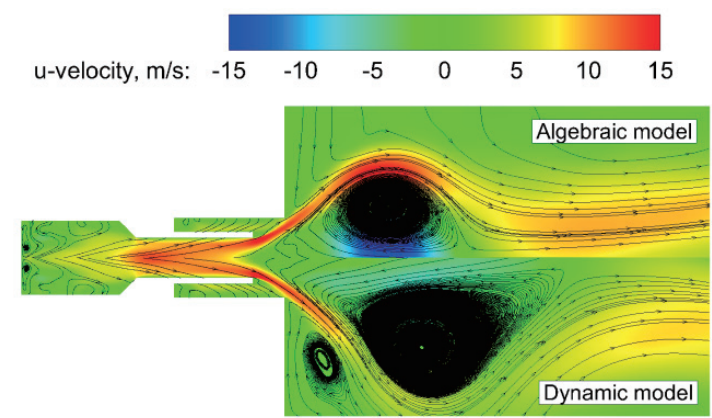

Fig. 7. Time Averaged u-Velocity Contours and Stream Lines; Algebraic Model (up) and Dynamic Model (down)

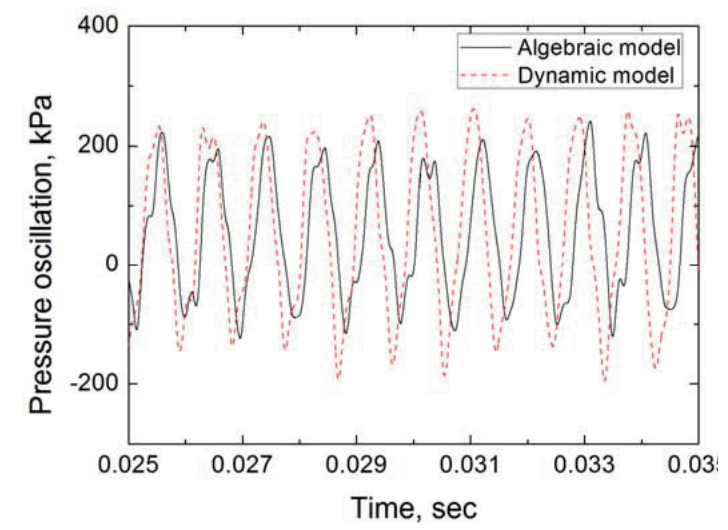

Fig. 8. Pressure Fluctuation inside the Oxygen Injector

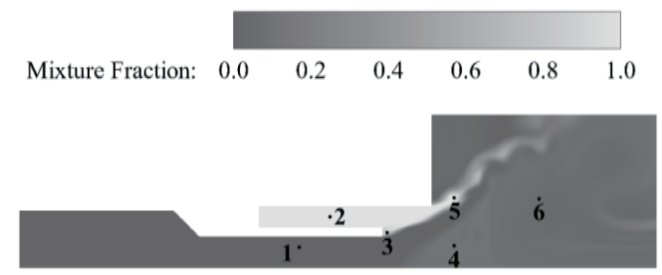

Fig. 9. Probe Location with Mixture Fraction compared with the algebraic SGS model [8].

A pressure oscillation in the injector causes instability in the mixing and combustion creating a requirement for accurate modeling. The dynamic SGS model is known to have a high accuracy in complex flow fields [8] such as that of turbulent mixing of the LRE injector. This is due to the calculation of the eddy viscosity in accordance with the local flow characteristics. The pressure fluctuations at probe 1 are compared with the SGS models in Fig. 8. A higher amplitude and faster oscillation in the dynamic SGS model over that of the algebraic SGS model is observed.

For a clearer understanding of this, a frequency analysis has been performed using the power spectrum densities calculation. The pressure fluctuation and PSD analysis are performed to analyze the dynamic characteristics of the flow structure. Fig. 9 shows the locations of the pressure measurements: inner side of injector (probe 1 and 2), near the LOx post (probe 3), phase-change region (probe 4), mixing layer (probe 5), and front of the CTRZ (probe 6).

Figure 10 represents the power spectral densities (PSD) of the pressure fluctuations at each location. The lower frequency, $1.05-1.08 \mathrm{kHz}$, is dominant in the LOx injector while the higher frequency is dominantly observed in the kerosene injector. Overall, the dominant frequencies are similar in both cases, but the frequencies of the dynamic model tend to be faster. The amplitude of the dynamic model is larger inside the injector even though the difference may disappear downstream. The dynamic model can capture the smaller scale eddies due to the calculation of the turbulent viscosity using the local flow properties. To compare the mixing level on the SGS model, the mixing efficiency is calculated using the following formula:
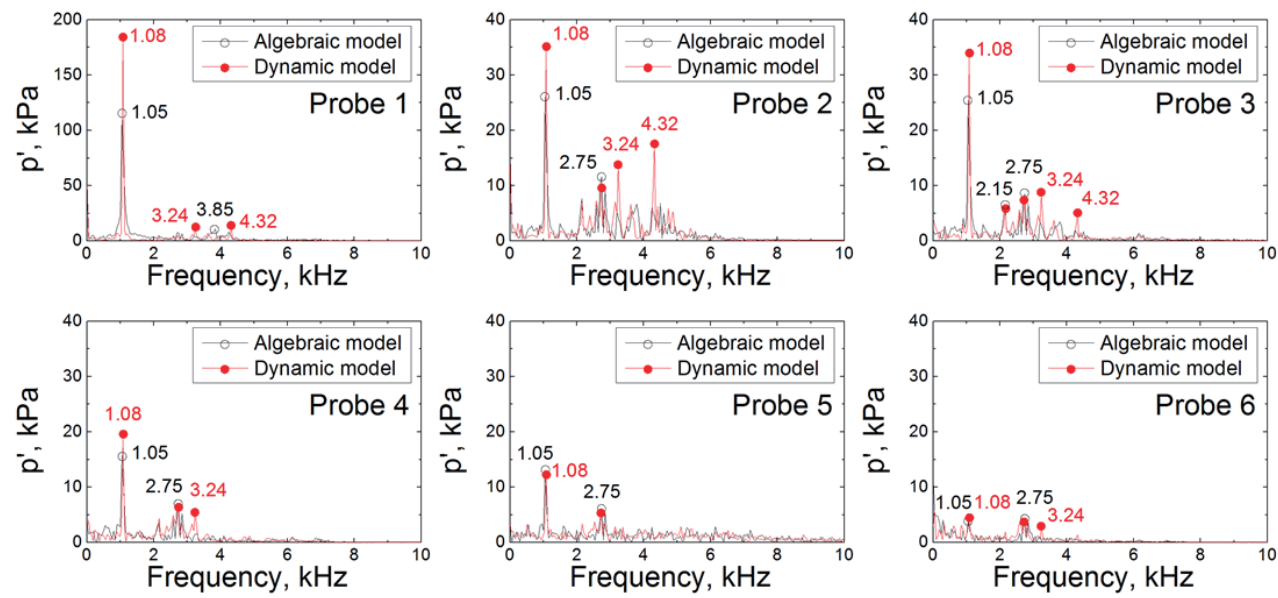

Fig. 10. PSD of Pressure Fluctuations on SGS Models 


$$
\begin{aligned}
& \eta_{\text {mix }}=\frac{\int \rho Y_{\text {Fuel }} u \alpha d A}{\int \rho Y_{\text {Fuel }} u d A} \\
& \alpha=\left(\begin{array}{c}
1 \quad\left(\phi_{\text {local }} \leq \phi_{\text {global }}\right) \\
\frac{1}{\phi_{\text {local }} / \phi_{\text {global }}}\left(\phi_{\text {local }}>\phi_{\text {global }}\right)
\end{array}\right.
\end{aligned}
$$

where $\mathrm{Y}_{\text {Fuel }}$ is the mass fraction of kerosene. The parameter $\alpha$ is determined by relating the local equivalence ratio, $\phi_{\text {local }}$ to the global equivalence ratio, $\phi_{\text {glocal }}$. Therefore, local mixing efficiency is less than 1 when the local equivalence ratio is larger than the global equivalence ratio. The mixing efficiency represents how much fuel is spread into the combustion chamber. Fig. 11 shows the time-averaged mixing efficiency. In both models, the efficiency increases as it moves downstream after the LOx post, where the mixing starts. The dynamic model shows it reaches perfect mixing at a slightly earlier state. The increase of the mixing efficiency in the dynamic model can be explained by the effect of the small scale turbulence eddy obtained from local turbulent flow dynamics.

Figure 12 represents distributions of the power density

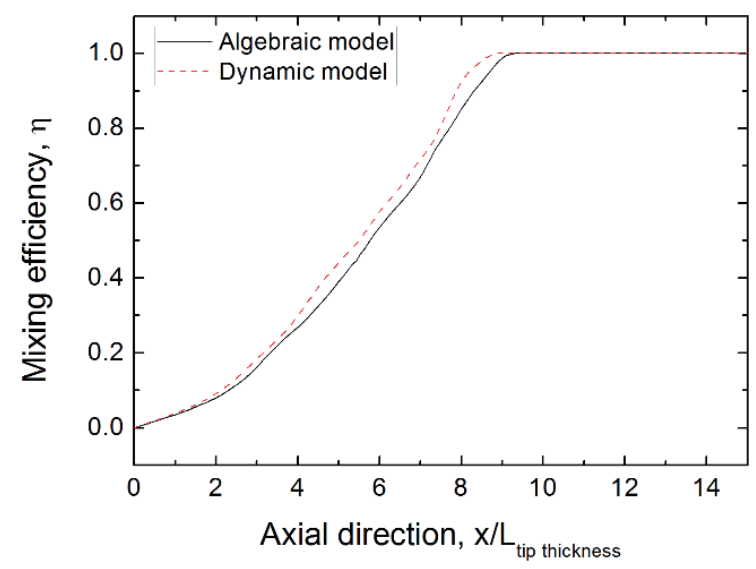

Fig. 11. Mixing Efficiency of the averaged flow-field on SGS Models

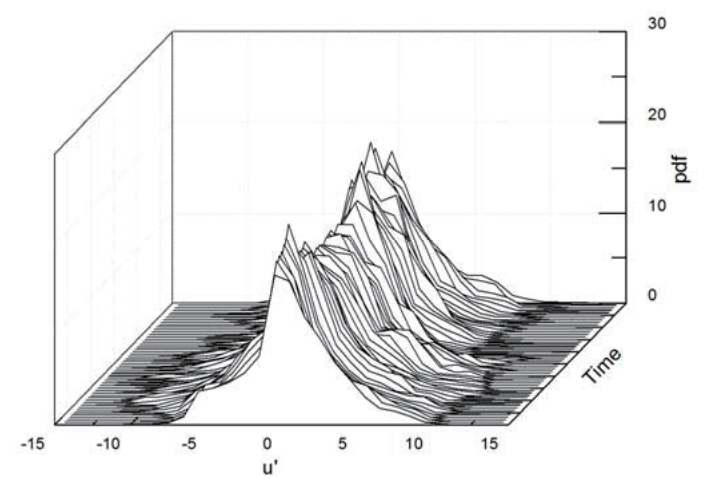

(a) Algebraic SGS model function (PDF) of $\mathrm{u}$-velocity perturbation in the chamber over an operation time. Both models provide a Gaussian distribution. In the combustion chamber, where there is a mixing zone by the swirling flow, the vortex is generated actively, which produces a wider range of velocity perturbation in the dynamics model. So, the dynamic model can capture more small eddies, resulting in better mixing.

Figure 13 shows the turbulent viscosity on an SGS model. Data is extracted from the boundary layer near the wall at the center of the oxidizer injector. The turbulent viscosity of the dynamic model has a large fluctuation compared to that of the algebraic model. In the dynamic model, the turbulent viscosity estimates negative values; which indicates a backscatter of kinetic energy. This phenomenon represents a reverse energy-transfer process from the subgrid to the supergrid scale which has a significant effect on the energy dissipation and generation activity near the wall.

This discrepancy in calculation affects the film thickness of the swirl injector due to a strong momentum occurring by the backscatter of kinetic energy. Fig. 14 shows the density contour comparison between the algebraic and dynamic model when the tangential velocity is twice the velocity of

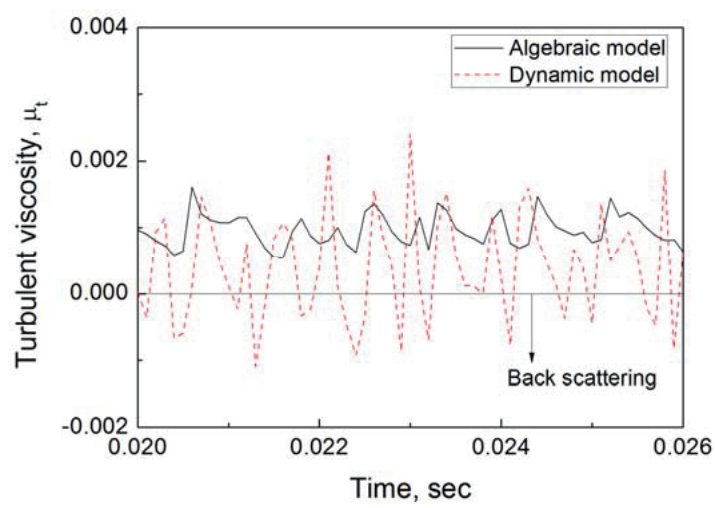

Fig. 13. History of Turbulent Viscosity at Position of Probe 1

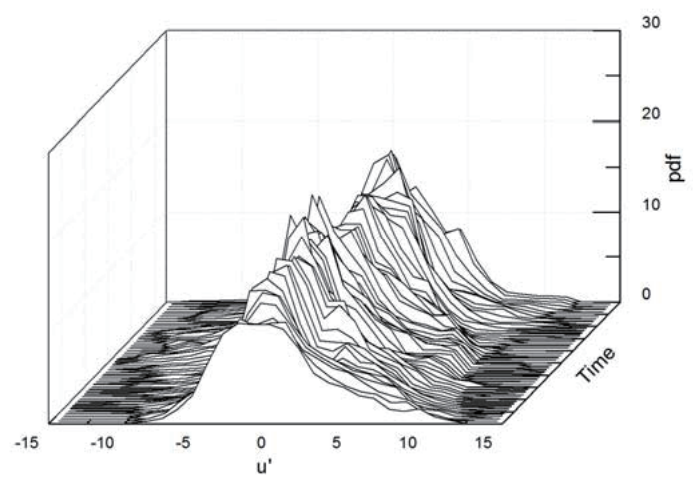

(b) Dynamic SGS model

Fig. 12. PDF of u-Velocity Perturbation in the Chamber 


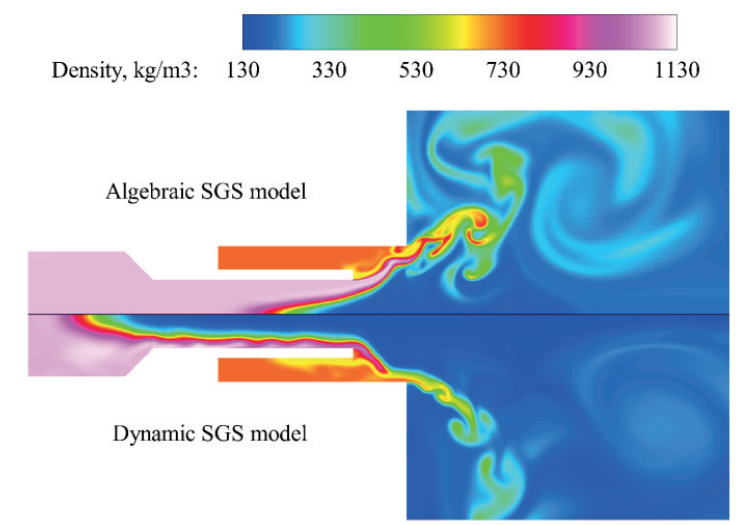

Fig. 14. Instantaneous Density Contour on SGS Models (Tangential Velocity: $\mathbf{x 2 )}$

the baseline case. The dynamic model's film is thinner than the algebraic model's result, leading to the creation of a long gaseous core in the liquid oxygen injector.

\section{Conclusions}

A numerical simulation has been performed to investigate the effect of the sub-grid scale models for large eddy simulation. The supercritical mixing characteristics of the swirl injector are discussed in this study. The RK-PR model is applied to calculate the real-fluid properties of kerosene and oxygen. The dynamic SGS model is compared with the classical algebraic SGS model. In terms of flow structure, the dynamic SGS produces a larger size of the CTRZ and a wider range of eddies, resulting in a faster flow fluctuation. The mixing efficiency is then analyzed to compare the level of mixing on SGS models. The dynamic model reaches perfect mixing at a slightly earlier state since it has a wide range of turbulent viscosities. To analyze the statistical quantities, the PDF of the u-velocity fluctuation is compared between two models. The dynamic SGS model generates a wider range of u-velocity perturbation. To assess the detailed characteristics of the dynamic SGS model, the turbulent viscosity is evaluated near the wall at the center of the oxidizer injector. In the dynamic SGS model, the turbulent viscosity has negative values referred to as a backscatter of kinetic energy which is an attractive characteristic of the turbulent kinetic energy transfer. In addition, the film thickness of the dynamic SGS model is thinner than that in the algebraic SGS model because the swirl momentum is maintained. As a result, the dynamic SGS model provides a more reasonable turbulent energy transfer and turbulent flow structures of a co-axial swirl injector under high-pressure condition.

\section{Acknowledgment}

This work was supported by Advanced Research Center Program (NRF-2013R1A5A1073861) through the National Research Foundation of Korea(NRF) grant funded by the Korea government(MSIP) contracted through Advanced Space Propulsion Research Center at Seoul National University. This research was partially supported by National Space Laboratory (NSL) Program (No. 2008-2006287) through the National Research Foundation of Korea (NRF) funded by the Ministry of Education, Science and Technology.

\section{References}

[1] V. Yang, "Modeling of supercritical vaporization, mixing, and combustion processes in liquid-fueled propulsion systems", Proceedings of the Combustion Institute, Vol. 28, 2000, pp. 925-942.

[2] J.C. Oefelein, "Thermophysical characteristics of shear coaxial LOx-H2 flames at supercritical pressure", Proceedings of the Combustion Institute, Vol. 30, 2005, pp. 2929-2937.

[3] Zong, N. and Yang, V., "Cryogenic Fluid Dynamics of Pressure Swirl Injectors at Supercritical Conditions", Physics of Fluids, Vol. 20, No. 5, 2008, 056103-1-14.

[4] M.G.D. Giorgi, A. Leuzzi, "CFD simulation of mixing and combustion in LOx/CH4 spray under supercritical conditions", 39th AIAA Fluid Dynamics Conference, No. 20094038, San Antonio, Texas, USA, 2009.

[5] Wang, Y. Z., Hua, Y. X. and Meng, H., "Numerical Studies of Supercritical Turbulent Convective Heat Transfer of Cryogenic Propellant Methane", Journal of Thermophysics and Heat Transfer, Vol. 24, No. 3, 2010, pp. 490-500.

[6] Lilly, D. K., "On the application of the eddy viscosity concept in the inertial subrange of turbulence", National Center for Atmospheric Research, Manuscript 123, 1966.

[7] Lilly, D. K., "The representation of small-scale turbulence in numerical simulations", Proc. IBM Scientific Computing Symp. on Environmental Sciences, Yorktown Heights, NY, 1967, pp. 195-210.

[8] M. Germano, U. Piomelli, P. Moin, W.H. Cabot, "A dynamic subgrid-scale eddy viscosity model", Physics of Fluids, Vol. 3, 1991, pp. 1760-1765.

[9] F. A. Jaberri, and P. J. Coucci, "Large eddy simulation of heat and mass transport in turbulent flows. Part 1: velocity field", International Journal of Heat and Mass Transfer, Vol. 46, 2003, pp. 1811-1825.

[10] A. Jammalamadaka, Z. Li, F. A. Jaberi, "Subgrid-scale models for large-eddy simulations of shock-boundary-layer interactions", AIAA Journal, Vol. 51, No. 5, 2013, pp. 1174-1188. 
[11] J. O'Brien, J. Urzay, M. Ihme, P. Moin, A. Saghafian, "Subgrid-scale backscatter in reacting and inert supersonic hydrogen-air turbulent mixing layers", J. Fluid Mech., Vol. 743, 2014, pp. 554-584.

[12] D. K. Lilly, "A proposed modification of the Germano subgrid-scale closure method", Physics of Fluids, Vol. 4, 1992, pp. 633-635.

[13] Soave, G., "Equilibrium Constants from a Modified Redlich-Kwong Equation of State", Chemical Engineering Science, Vol. 27, No. 6, June, 1972, pp.1197-1203

[14] Peng, D. and Robinson D. B., "A New Two-Constant Equation of State", Industry Engineering Chemical Fundamentals, Vol. 15, No. 1, February 1976, pp.59-64

[15] M. Cismondi, and J. Mollerup, "Development and application of a three-parameter RK-PR equation of state", Fluid Phase Equilibria, Vol. 232, 2005, pp. 74-89.

[16] S. K. Kim, H. S. Choi, and Y. M. Kim, “Thermodynamic Modeling Based on a Generalized Cubic Equation of State for Kerosene/LOx Rocket Combustion", Combustion and Flame, Vol. 159, Issue 3, 2012, pp. 1351-1365.

[17] J. Y. Heo, Numerical Study on Supercritical Mixing and Combustion Characteristics of a Kerosene/LOx Co-axial Swirl Injector, Ph.D. Thesis, Korea Aerospace University, 2015.

[18] T. Chung, M. Ajlan, L.L. Lee, and K.E. Starling, "Generalized multiparameter correlation for nonpolar and polar fluid transport properties", Industrial \& Engineering
Chemistry Research, Vol. 27, 1988, pp. 671-679.

[19] S. Takahashi, "Preparation of a generalized chart for the di_usion coe_cients of gases at high pressures", Japanese J. of Chem. Eng., Vol. 7, No. 6, 1974, pp. 417-420.

[20] H. Meng, and V. Yang, "A Unified Treatment of General Fluid Thermodynamics and Its Application to a Preconditioning Scheme", J. Computational Physics, Vol. 189, No. 1, 2003, pp. 277-304.

[21] J. C. Kim, K. H. Yoo, and H. G. Sung, "Large-eddy Simulation and Acoustic Analysis of a Turbulent Flow Field in a Swirl-stabilized Combustor", J. Mechanical Science and Technology, Vol. 25, 2011, pp. 2703-2710.

[22] K. H. Yoo, J. C. Kim, and H. G. Sung, "Effects of Cooling Flow on the Flow Structure and Acoustic Oscillation in a Swirl-stabilized Combustor, Part I. Flow Characteristics", J. Visualization, Vol. 16, 2013, pp. 287-295.

[23] H. G. Sung, K. H. Yoo, and J. C. Kim, "Effects of Cooling Flow on the Flow Structure and Acoustic Oscillation in a Swirl-stabilized Combustor, Part II. Acoustic Analysis", J. Visualization, Vol. 17, 2014, pp. 69-76.

[24] H. S. Choi, “Technical Report”, Korea Aerospace Research Institute, 2010.

[25] N. Zong, Modeling and Simulation of Cryogenic Fluid Injection and Mixing Dynamics Under Supercritical Conditions, Ph.D. Thesis, The Pennsylvania State University, 2005. 